\title{
Microneedles for drug and vaccine delivery and patient monitoring
}

\author{
Ryan Donnelly $^{1} \cdot$ Dennis Douroumis ${ }^{2}$
}

Published online: 30 July 2015

(C) Controlled Release Society 2015

Despite the limited number of drugs amenable to administration across the skin, due to the excellent barrier properties of the stratum corneum, the worldwide transdermal product market is worth approximately $\$ 32$ billion per year. The major growth drivers are greater patient acceptance leading to wider market penetration, lowered development costs and introduction of novel technologies instigating market growth. The cost of new drug discovery has spiralled to around $\$ 800$ million and time to market can be 13 to 15 years. However, transdermal delivery development of existing drugs costs less (approx $\$ 10$ to $\$ 15$ million) and involves a shorter timeline (4 to 8 years), enabling improved product lifecycle management by extending IP protection. As industry increasingly recognises the market potential for transdermal delivery, R\&D efforts have increased, leading to an influx of new technologies aimed at increasing the range of drug types deliverable transdermally. A key market area for development is transdermal delivery of therapeutic peptides and proteins. There are over 100 biotechnology-derived medicines marketed, covering nine major therapeutic areas, now representing approximately $15 \%$ of all prescriptions. Today, more than 4000 companies worldwide are involved in biotechnology research, with total revenue increasing from $\$ 7.7$ billion in 1993 to around $\$ 103$ billion in 2013. A major challenge to successful clinical use of these hydrophilic, high molecular weight "biotech" molecules is drug delivery. Due to enzymatic breakdown and poor GI absorption, the parenteral route is presently the only option.

Ryan Donnelly

R.Donnelly@qub.ac.uk

School of Pharmacy, Queen's University, Belfast, UK

2 Department of Pharmaceutical, Chemical, and Environmental Sciences, University of Greenwich, London, UK
Microneedles, minimally invasive devices that painlessly, and without drawing blood penetrate the skin's stratum corneum barrier, have the potential to effectively overcome this problem. Thus, such systems could greatly enhance market size by removing dependence of efficient transdermal transport on drug physicochemical properties, allowing a much greater range of drugs to be delivered transdermally. In fact, microneedle arrays have already successfully delivered oligonucleotides, desmopressin, DNA, vaccines, insulin and human growth hormone in vivo. Indeed, a report from Greystone Associates, Microneedles in Medicine: Technology, Devices, Markets and Prospects, put the potential market for microneedle drug delivery systems at just under $\$ 400$ million globally in 2012. Since microneedles are frequently targeted not only to the $\$ 32$ billion transdermal drug delivery and $\$ 25$ billion global vaccine markets but also to the $\$ 120$ billion global biologics market, significant further growth is anticipated.

The future appears to be very bright for new delivery and, potentially, monitoring/diagnostic systems based upon microneedle technologies. The ever-increasing amount of fundamental knowledge appears to be feeding industrial development. Microneedles have many advantages over conventional needle-and-syringe-based delivery systems for biological agents, in particular, in terms of reduced pain, infection risk and the ability to control administration. Skin barrier function disturbance is minimal and recovery rapid. Once regulatory hurdles are overcome and manufacturing processes developed, optimised and validated to current good manufacturing practice standards, the benefits for patients, and ultimately for the industry, will be considerable.

Given the inherent safety features of microneedle systems, it is easy to foresee a time within the next 10 years when vaccination programmes in the developing world are based around microneedles. The fact that most microneedle 
formulate biomolecules, such as vaccine antigens in the dry state, the cold chain will be circumvented. Needlestick injuries also will be obviated. Such an intervention could massively improve the quality of life, life expectancy and economic productivity of developing countries. Accordingly, the potential impact of $\mathrm{MN}$ research and ultimate commercialisation is vast. Once vaccine products are accepted by regulators, healthcare providers and patients, other microneedle-based products for everyday patient and consumer use will become widely used to the benefit of the pharmaceutical, medical devices and cosmetics industries and patients worldwide.
In this Special Issue of Drug Delivery and Translational Research, there is a focus on novel applications of microneedles in drug, vaccine and gene delivery, as well as in minimally invasive monitoring/diagnosis. Many of the leading groups in the field have contributed papers. It is our hope that, by showcasing the many applications of microneedles in one place here, interest in and knowledge of microneedles in the broader drug delivery field will be enhanced and industrial interest will increase, leading, in due course, to patient and economic benefits. 